\title{
Temporal trends and demographic variations in hospitalizations with angiodysplasia of the intestine: A U.S. population based study
}

Shantanu Devanand Solanki ${ }^{1}$, Khwaja Fahad Haq ${ }^{2}$, Zahava Farkas ${ }^{3}$, Zubair Khan ${ }^{4}$, Raja Chandra Chakinala ${ }^{3}$, Alina Kifayat ${ }^{3}$, Muhammad Ali Khan ${ }^{5}$, Abhishek Bhurwal ${ }^{6}$, Christopher Nabors ${ }^{3}$, Edward Lebovics ${ }^{3}$

\begin{abstract}
Introduction: Angiodysplasia is the most common vascular lesion of the gastrointestinal (Gl) tract. However, limited epidemiological data exists on hospitalizations related to angiodysplasia of the intestine (ADOI) in the United States (U.S.). The aim of this study is to analyze inpatient trends and outcomes in hospitalizations with ADOI.

Methods: We analyzed the National Inpatient Sample (NIS) database for all subjects with discharge diagnosis of angiodysplasia of the intestine with or without hemorrhage (ICD-9 code 569.85 and 569.84 respectively) as principal and/or secondary diagnosis during the period from 2001 to 2011. Results: In 2001, there were 39,244 admissions with discharge diagnosis of ADOI as compared to 41,214 in 2011 ( $p<0.0001$ ). Women (58.0\%) were more commonly affected than men $(42.0 \%)(p<0.0001)$. Overall in-hospital mortality rate was $2.2 \%$ for patients with ADOI with hemorrhage and $1.6 \%$ for patients with ADOI without hemorrhage ( $p<0.0001)$. Mean cost of hospitalization increased from $\$ 10,701$ in 2001 to $\$ 12,478$ in 2011 (16.6\% increase, $p<0.0001$ ).

Conclusion: The total number of hospitalizations with ADOI increased significantly with women having significantly higher hospitalization rates. Mortality rate was higher in hospitalizations with ADOI with hemorrhage than those without hemorrhage. There was a notable increase in the average cost of hospitalization.
\end{abstract}

Keywords: angiodysplasia, gastrointestinal bleeding, endoscopy

\section{INTRODUCTION}

Angiodysplasia is the most common vascular lesion of the GI tract (1). The terms angiodysplasia, arteriovenous malformation, and vascular ectasia have been synonymously used to describe the same entity (2). It is responsible for close to $6.0 \%$ of cases with lower GI bleeding (3). However, epidemiological data on this very common reason of lower GI hemorrhage is limited. Therefore, we designed this study to determine the hospitalization rates of ADOI in the U.S. population, analyze trend in demographic variations, and outcomes, and evaluate its economic impact on the healthcare system.

\section{METHODS}

\section{Source of Data}

The NIS, designed by Agency for Healthcare Research and Quality (AHRQ), is the largest all-payer inpatient database in the U.S. Data are compiled yearly and contain discharge information from over 1200 hospitals located across 45 states in the U.S. The NIS was designed to approximate a $20 \%$ stratified sample of community hospitals in the country and provides sampling weights to calculate national estimates (4). The NIS contains information included in a typical

\footnotetext{
Guthrie Robert Packer Hospital, Sayre, PA, USA

2 Henry Ford Hospital, Detroit, MI, USA

3 New York Medical College at Westchester Medical Center, Valhalla, NY, USA

4 University of Toledo Medical Center, Toledo, OH, USA

5 University of Tennessee Health Science Center, Memphis, TN, USA

6 St. Barnabas Medical Center, Livingston, NJ, USA
}

Correspondence: Shantanu Devanand Solanki

Hospitalist Program, Guthrie Robert Packer Hospital, Sayre, PA, USA

E-mail: shan4788@gmail.com

Received: 9 July 2018, Accepted: 6 Aug 2018

(C) 2018 by the authors; licensee Modestum Ltd., UK. This article is an open access article distributed under the terms and conditions of the Creative Commons Attribution License (http://creativecommons.org/licenses/by/4.0/).

Electronic Journal of General Medicine 
discharge summary, with safeguards in place to protect the privacy of individual patients, physicians, and hospitals. Each individual hospitalization is de-identified and maintained in the NIS as a unique entry with one primary discharge diagnosis and approximately twenty-four secondary diagnoses during that hospitalization. Each entry also carries information on demographic details, insurance status, comorbidities, primary/secondary procedures, hospitalization outcomes, length of stay, and cost of care. The internal validity of the database is guaranteed by annual data quality assessments of the sample. The external validity of the sample is strengthened by comparison with databases like American Hospital Association (AHA) Annual Survey of Hospitals, National Hospital Discharge Survey from the National Center for Health Statistics, and Medicare Provider and Analysis Review (MedPAR) inpatient data from the Centers for Medicare and Medicaid Services $(5,6)$.

\section{Study Design}

This study was exempted from institutional review board (IRB) as it involved de-identified data. It is a cross-sectional study in which we queried the NIS database from the year 2001 to 2011 to identify all the hospitalizations with angiodysplasia of the intestine. We extracted data for all the hospitalizations from 2001 to 2011 with primary diagnosis or secondary diagnosis of angiodysplasia of the intestine with or without hemorrhage, which were identified by ICD-9 codes 569.85 and 569.84 respectively. Patients with age less than 18 years were excluded. Also, hospitalizations with missing information related to age, gender, admission/discharge date, and in-hospital mortality status and demographics and comorbidities were excluded as seen in previous well-designed studies $(7,8)$. NIS data were merged with cost-to-charge ratio (CCR) files available from the Healthcare Cost and Utilization Project (HCUP) to calculate estimated cost of hospitalizations. We estimated the cost of each inpatient stay by multiplying the total hospital charge with cost-to-charge ratio.

\section{Variables and Statistical Analysis}

SAS 9.4 (SAS Institute Inc., Cary, North Carolina, USA) was utilized for statistical analyses. Since NIS represents a 20\% stratified random sample of U.S. hospitals, analyses were performed using hospital-level discharge weights provided by the NIS, to obtain national estimates of hospitalizations. The frequency of hospitalizations was calculated for each year. We calculated hospitalizations related to angiodysplasia per 1 million U.S. population by dividing yearly hospitalizations by $20 \%$ of the U.S. census population more than 18 years of age for that year (9). These hospitalizations were also calculated in subgroups of age (18-34, 35-49, 50-64, 65-79, and >80 years), gender, race (White, Black, Hispanic, and Others), insurance status (Medicare/Medicaid, private insurance, and self-pay/other), hospital location in different U.S. regions (Northeast, Midwest, South, and West), bed size of the hospital (small, medium, and large), and teaching status of the hospital (urban teaching, urban non-teaching, and rural). According to AHRQ, a hospital is considered to be a teaching hospital if it is: a) an AMA-approved residency program, b) a member of the Council of Teaching Hospitals, or c) a hospital with a full-time intern and resident-to-bed (IRB) ratio more than 0.25 (10). HCUP provides a quartile classification of the estimated median household income of residents derived from ZIP Code-demographic data. The four quartiles, from 1 to 4 , indicate the poorest to wealthiest populations. The bed size varies by region and teaching status of the hospitals. The Cochrane-Armitage trend test was used to calculate trends in categorical variables.[11] The Wilcoxon rank sum test was used to assess continuous variables (12).

\section{RESULTS}

\section{Demographics}

Patient characteristics are summarized in Table 1. The number of hospitalizations with ADOI increased progressively from 39,244 admissions in 2001 as compared to 41,214 in 2011 ( $p$ < 0.0001) (Figure 1). A total of 432,452 hospitalizations with ADOI were reported during this time period. Out of these hospitalizations, $64.7 \%$ were without hemorrhage and $35.3 \%$ were with hemorrhage during the study period. These patients were predominantly White and in the 65 to 74 year age group. The mean age with standard deviation was $75.2 \pm 25.7$ years for the entire affected population. It was $74.2 \pm$ 26.1 years for men and $76 \pm 25.3$ years for women. Women were more affected than men during the study period (Figure 2) and accounted for a higher number of hospitalizations than men (58\% vs $42 \%)$. Most hospitalizations were seen in urban non-teaching hospitals (48.9\%) followed by urban teaching hospitals (38.9\%) and rural hospitals (11.9\%). Medicare paid for a staggering $81.8 \%$ of the total hospitalizations. Hospitals with large bed size accounted for $63.8 \%$ of the total hospitalizations whereas hospitals with small bed size accounted for $10.7 \%$. 
Table 1: Baseline characteristics of angiodysplasia hospitalizations

\begin{tabular}{|c|c|c|c|c|c|c|c|c|c|c|c|c|c|}
\hline Year & 2001 & 2002 & 2003 & 2004 & 2005 & 2006 & 2007 & 2008 & 2009 & 2010 & 2011 & OVERALL & P-VAL \\
\hline Number of obs. (n) & 39,244 & 38,297 & 37,122 & 39,048 & 40,336 & 38,518 & 37,749 & 41,068 & 41,029 & 38,827 & 41,214 & 432,452 & $<0.0001$ \\
\hline \multicolumn{14}{|l|}{ Hemorrhage (\%) } \\
\hline Yes & 32.1 & 32.1 & 32.3 & 30.5 & 29.8 & 34.0 & 36.3 & 36.6 & 42.3 & 40.4 & 40.8 & 35.3 & $<0.0001$ \\
\hline No & 67.9 & 67.9 & 67.7 & 69.5 & 70.2 & 66.0 & 63.7 & 63.4 & 57.7 & 59.7 & 59.2 & 64.7 & \\
\hline \multicolumn{14}{|l|}{ Age in years (\%) } \\
\hline $18-34$ & 0.4 & 0.5 & 0.6 & 0.8 & 0.5 & 0.5 & 0.5 & 0.5 & 0.5 & 0.8 & 0.7 & 0.6 & $<0.0001$ \\
\hline $35-49$ & 2.5 & 2.3 & 2.3 & 2.7 & 2.7 & 2.5 & 2.4 & 2.6 & 3.0 & 2.8 & 2.0 & 2.5 & 0.127 \\
\hline $50-64$ & 10.2 & 10.9 & 11.5 & 11.6 & 12.5 & 13.4 & 11.7 & 13.3 & 14.0 & 13.7 & 15.3 & 12.6 & $<0.0001$ \\
\hline $65-79$ & 45.3 & 45.0 & 45.9 & 44.2 & 43.0 & 43.0 & 44.0 & 41.8 & 42.5 & 43.1 & 41.5 & 43.5 & $<0.0001$ \\
\hline$>=80$ & 41.5 & 41.2 & 39.6 & 40.6 & 41.1 & 40.5 & 41.2 & 41.6 & 40.1 & 39.6 & 40.5 & 40.7 & 0.0008 \\
\hline \multicolumn{14}{|l|}{ Gender (\%) } \\
\hline Male & 41.4 & 39.7 & 39.7 & 40.9 & 41.0 & 42.7 & 42.3 & 43.3 & 43.4 & 43.2 & 44.6 & 42.0 & $<0.0001$ \\
\hline Female & 58.6 & 60.3 & 60.3 & 59.1 & 59.0 & 57.3 & 57.7 & 56.7 & 56.7 & 56.9 & 55.4 & 58.0 & \\
\hline \multicolumn{14}{|l|}{ Race (\%) } \\
\hline White & 61.1 & 57.9 & 57.4 & 57.7 & 59.6 & 59.2 & 55.2 & 61.6 & 65.7 & 67.8 & 67.5 & 61.1 & $<0.0001$ \\
\hline Black & 8.3 & 9.1 & 8.9 & 9.3 & 7.2 & 9.1 & 10.0 & 10.4 & 9.3 & 12.9 & 13.5 & 9.8 & $<0.0001$ \\
\hline Hispanic & 5.2 & 5.8 & 6.2 & 4.4 & 5.5 & 6.7 & 6.0 & 5.4 & 6.9 & 6.1 & 6.6 & 5.9 & 0.4758 \\
\hline Others & 1.7 & 2.0 & 2.6 & 2.6 & 2.5 & 2.9 & 2.7 & 3.5 & 3.9 & 3.4 & 3.3 & 2.8 & $<0.0001$ \\
\hline \multicolumn{14}{|l|}{ Region (\%) } \\
\hline Northeast & 22.5 & 22.8 & 21.8 & 20.4 & 22.4 & 21.5 & 20.5 & 20.5 & 19.8 & 21.6 & 21.0 & 21.3 & $<0.0001$ \\
\hline Midwest & 24.5 & 21.8 & 25.8 & 26.4 & 23.9 & 24.3 & 27.5 & 24.8 & 25.8 & 25.0 & 26.3 & 25.1 & $<0.0001$ \\
\hline South & 38.9 & 39.2 & 36.6 & 38.1 & 37.3 & 37.9 & 36.6 & 38.9 & 39.8 & 39.6 & 37.7 & 38.2 & 0.0064 \\
\hline West & 14.2 & 16.2 & 15.8 & 15.1 & 16.5 & 16.3 & 15.5 & 15.8 & 14.6 & 13.8 & 14.9 & 15.3 & $<0.0001$ \\
\hline \multicolumn{14}{|l|}{ Location (\%) } \\
\hline Rural & 13.6 & 12.7 & 15.9 & 11.4 & 11.9 & 9.9 & 11.4 & 11.1 & 12.3 & 11.5 & 9.5 & 11.9 & $<0.0001$ \\
\hline Urban nonteaching & 49.4 & 49.1 & 47.5 & 51.1 & 55.6 & 49.8 & 47.7 & 48.5 & 46.4 & 47.5 & 44.9 & 48.9 & $<0.0001$ \\
\hline Urban teaching & 37.0 & 38.2 & 36.5 & 37.5 & 32.5 & 40.2 & 40.6 & 40.4 & 40.0 & 40.2 & 44.6 & 38.9 & $<0.0001$ \\
\hline \multicolumn{14}{|c|}{ Median Household Income (\%) } \\
\hline Quartile 1 & 6.4 & 4.9 & 25.4 & 24.8 & 24.1 & 24.5 & 26.7 & 26.3 & 25.9 & 27.1 & 27.6 & 22.2 & $<0.0001$ \\
\hline Quartile 2 & 23.7 & 19.5 & 26.8 & 27.0 & 25.3 & 24.6 & 26.4 & 27.5 & 26.9 & 25.8 & 24.4 & 25.3 & $<0.0001$ \\
\hline Quartile 3 & 26.4 & 25.1 & 24.8 & 23.5 & 26.3 & 25.3 & 23.8 & 23.6 & 24.1 & 24.5 & 25.9 & 24.8 & $<0.0001$ \\
\hline Quartile 4 & 42.3 & 48.3 & 21.1 & 23.0 & 22.6 & 23.7 & 21.5 & 21.4 & 21.4 & 21.0 & 20.7 & 26.0 & $<0.0001$ \\
\hline \multicolumn{14}{|l|}{ Payment (\%) } \\
\hline Medicare & 82.8 & 83.3 & 83.5 & 81.6 & 82.9 & 82.2 & 82.1 & 80.2 & 79.3 & 80.5 & 81.9 & 81.8 & $<0.0001$ \\
\hline Medicaid & 2.9 & 3.0 & 3.2 & 3.0 & 3.2 & 3.6 & 2.9 & 3.2 & 4.0 & 4.4 & 3.7 & 3.4 & $<0.0001$ \\
\hline Private insurance & 12.0 & 11.9 & 11.4 & 12.8 & 11.6 & 11.3 & 12.9 & 14.2 & 13.8 & 12.4 & 11.5 & 12.4 & $<0.0001$ \\
\hline Others (includes self-pay) & 2.2 & 1.8 & 1.8 & 2.7 & 2.3 & 2.6 & 2.1 & 2.5 & 2.8 & 2.5 & 2.6 & 2.4 & $<0.0001$ \\
\hline \multicolumn{14}{|l|}{ Bed size } \\
\hline Small & 10.7 & 10.5 & 10.0 & 9.3 & 10.4 & 12.5 & 10.5 & 11.2 & 11.3 & 10.0 & 11.4 & 10.7 & $<0.0001$ \\
\hline Medium & 26.4 & 25.4 & 25.6 & 26.4 & 24.5 & 26.0 & 27.9 & 23.8 & 22.5 & 24.2 & 24.6 & 25.2 & $<0.0001$ \\
\hline Large & 63.0 & 64.1 & 64.2 & 64.3 & 65.1 & 61.4 & 61.3 & 65.0 & 64.8 & 65.0 & 63.0 & 63.8 & $<0.0001$ \\
\hline In-hospital Mortality (\%) & 2.1 & 2.5 & 2.0 & 1.8 & 1.9 & 2.0 & 1.8 & 1.7 & 2.1 & 2.1 & 2.1 & 2.0 & 0.02 \\
\hline
\end{tabular}

\section{AHRQ comorbidity measures (\%)}

\begin{tabular}{lccccccccccccc}
\hline Obesity & $\#$ & 2.0 & 2.3 & 2.5 & 3.2 & 3.7 & 4.6 & 6.4 & 6.8 & 7.5 & 8.7 & 4.4 & $<0.0001$ \\
\hline Hypertension & $\#$ & 38.0 & 47.5 & 54.0 & 54.5 & 59.4 & 61.9 & 64.6 & 66.1 & 68.7 & 71.1 & 53.5 & $<0.0001$ \\
\hline Diabetes mellitus & $\#$ & 24.9 & 24.9 & 26.7 & 28.6 & 30.9 & 31.6 & 32.1 & 34.8 & 35.4 & 36.8 & 28.0 & $<0.0001$ \\
\hline Congestive heart failure & $\#$ & 22.1 & 22.2 & 24.8 & 25.2 & 25.0 & 24.5 & 25.0 & 24.4 & 25.8 & 27.1 & 22.4 & $<0.0001$ \\
\hline Chronic pulmonary disease & $\#$ & 23.9 & 24.7 & 26.1 & 28.4 & 27.7 & 28.7 & 27.9 & 28.5 & 30.4 & 30.0 & 25.2 & $<0.0001$ \\
\hline Peripheral vascular disease & $\#$ & 7.2 & 7.4 & 8.1 & 9.0 & 10.3 & 11.6 & 12.6 & 13.8 & 14.1 & 15.6 & 10.1 & $<0.0001$ \\
\hline Renal failure & $\#$ & 24.4 & 24.4 & 27.8 & 31.0 & 37.5 & 39.2 & 39.7 & 43.2 & 46.6 & 49.1 & 33.1 & $<0.0001$ \\
\hline Neurological disorders & $\#$ & 6.7 & 6.6 & 6.9 & 6.8 & 7.4 & 8.2 & 8.4 & 9.1 & 9.3 & 9.5 & 7.2 & $<0.0001$ \\
\hline Anemia & $\#$ & 24.1 & 23.9 & 26.1 & 27.6 & 29.2 & 30.4 & 30.8 & 35.3 & 33.9 & 36.2 & 27.1 & $<0.0001$ \\
\hline Weight loss & $\#$ & 2.2 & 2.1 & 2.8 & 2.8 & 3.4 & 3.7 & 4.4 & 5.4 & 5.9 & 6.9 & 3.6 & $<0.0001$ \\
\hline Rheumatic disorders & $\#$ & 2.5 & 2.5 & 2.8 & 3.0 & 3.4 & 3.6 & 3.4 & 3.9 & 3.9 & 3.9 & 3.0 & $<0.0001$ \\
\hline Psychiatric disorders & $\#$ & 6.3 & 6.7 & 7.7 & 8.9 & 9.0 & 9.4 & 10.8 & 13.1 & 13.3 & 14.4 & 9.1 & $<0.0001$ \\
\hline Liver disease & $\#$ & 4.0 & 4.3 & 5.0 & 5.0 & 5.2 & 5.2 & 5.4 & 6.8 & 6.3 & 7.9 & 5.0 & $<0.0001$ \\
\hline AHRQ inicates Agency & & & & & & & & & & & & &
\end{tabular}

AHRQ indicates Agency for Healthcare Research and Quality.

Variables are AHRQ comorbidity measures.

Neurological disorders include hemiplegia, paralysis, and others.

Psychiatric disorders include depression, psychosis, and others.

Rheumatic disorders include rheumatoid arthritis and other collagen vascular disorders

${ }^{\#}$ Of note, data related to comorbidity measures were available only from 2002 to 2010. 


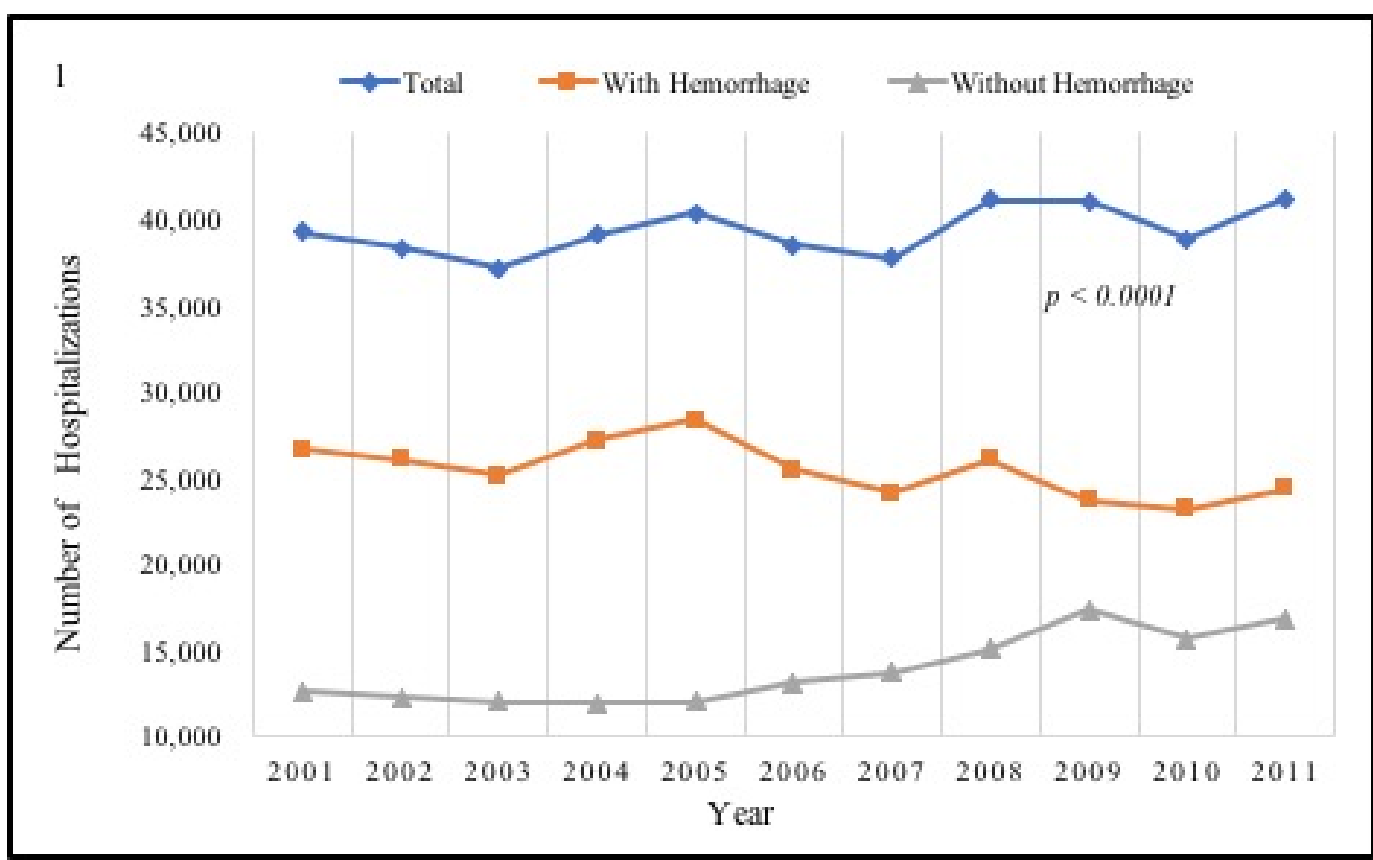

Figure 1: Total number of ADOI hospitalizations, $A D O I$ hospitalizations with hemorrhage, and ADOI hospitalizations without hemorrhage

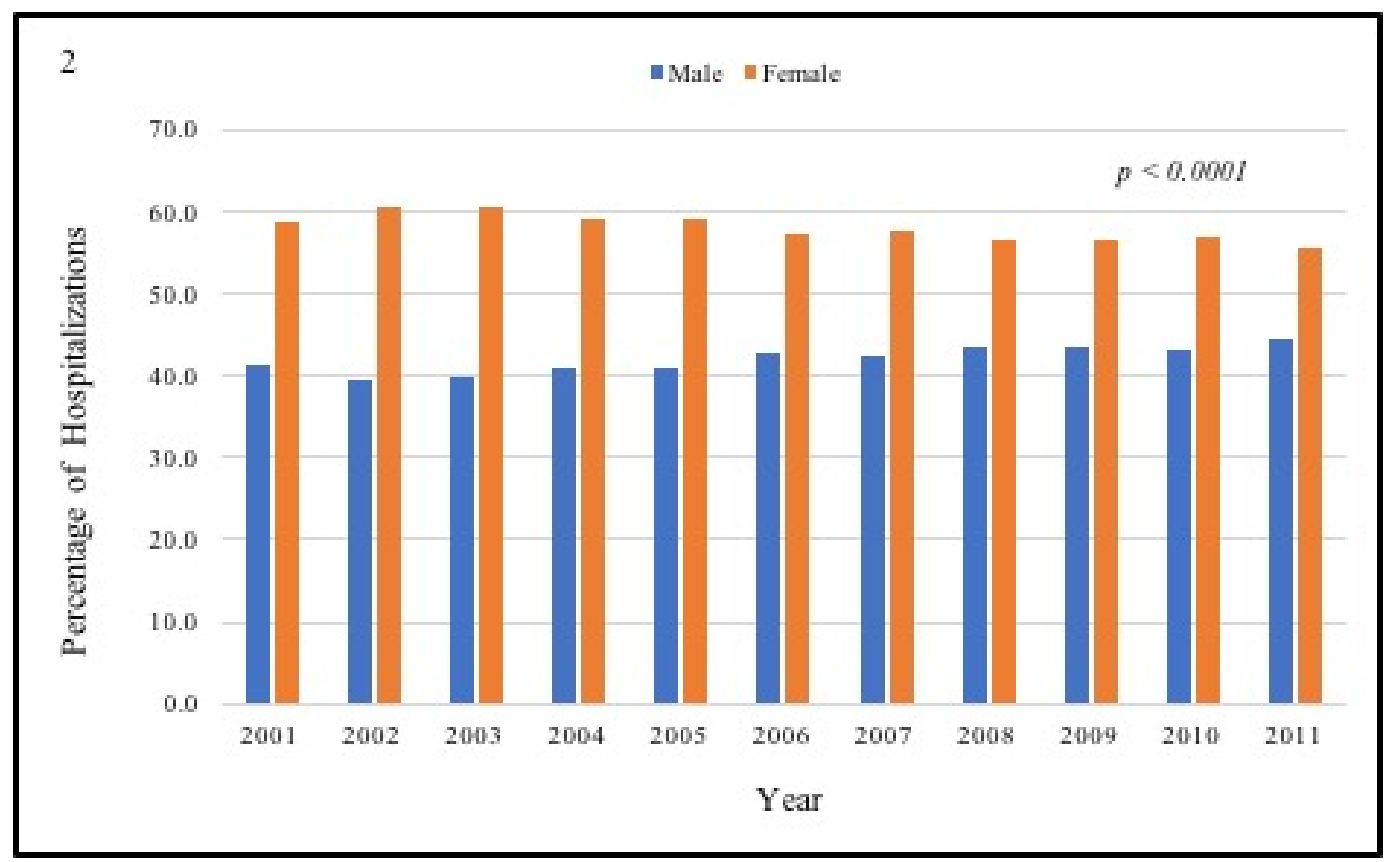

Figure 2: Gender-wise distribution of ADOI hospitalizations

\section{Trends in Hospitalizations}

The hospitalization rate increased significantly from 185 per 1 million U.S. population in 2001 to 867 per 1 million U.S. population in 2011 (369.1\% increase; $p<0.0001$; Table 2). The rate of hospitalization for ADOI with hemorrhage increased from 125 to 513 per million U.S. population per year (309\% increase; $p<0.0001)$. Also, the rate of hospitalization for ADOI without hemorrhage increased from 59 to 354 per million U.S. population per year (497\% increase; $p<0.0001$ ). The hospitalization rate increased significantly for all age groups $(p<0.0001)$. The hospitalization rate was higher in White race throughout the study period. Although, the hospitalization rate per million population was higher in women compared to men every single year during the study period (Table 2), the percent increase in hospitalizations was higher in men compared to women ( $405 \%$ versus $343 \% ; p$ <0.0001). Midwest region witnessed the highest percent increase in hospitalization per million population and Northeast witnessed the lowest rate $(404 \%$ vs $339 \% ; p<0.0001)$. 
Table 2: Angiodysplasia hospitalizations per 1 million US population

\begin{tabular}{|c|c|c|c|c|c|c|c|c|c|c|c|c|c|c|}
\hline Year & 2001 & 2002 & 2003 & 2004 & 2005 & 2006 & 2007 & 2008 & 2009 & 2010 & 2011 & Average & $\begin{array}{l}\text { Percent } \\
\text { change }\end{array}$ & P-value \\
\hline Overall & 185 & 892 & 855 & 889 & 908 & 857 & 831 & 893 & 882 & 828 & 867 & 808 & 369.1 & $<0.0001$ \\
\hline \multicolumn{15}{|l|}{ Hemorrhage } \\
\hline Yes & 125 & 606 & 579 & 618 & 638 & 566 & 530 & 566 & 509 & 494 & 513 & 522 & 308.8 & $<0.0001$ \\
\hline No & 59 & 286 & 276 & 271 & 270 & 292 & 301 & 327 & 373 & 334 & 354 & 286 & 496.5 & $<0.0001$ \\
\hline \multicolumn{15}{|l|}{ Age in years } \\
\hline $18-34$ & 1 & 4 & 5 & 7 & 5 & 4 & 4 & 5 & 4 & 6 & 6 & 5 & 638.5 & $<0.0001$ \\
\hline $35-49$ & 5 & 21 & 20 & 24 & 24 & 22 & 20 & 23 & 26 & 23 & 17 & 20 & 275.0 & $<0.0001$ \\
\hline $50-64$ & 19 & 97 & 98 & 103 & 113 & 115 & 97 & 119 & 123 & 113 & 132 & 103 & 599.4 & $<0.0001$ \\
\hline $65-79$ & 84 & 401 & 393 & 393 & 391 & 369 & 366 & 373 & 375 & 357 & 360 & 351 & 329.8 & $<0.0001$ \\
\hline$>=80$ & 77 & 368 & 339 & 361 & 374 & 347 & 342 & 372 & 353 & 328 & 351 & 328 & 358.0 & $<0.0001$ \\
\hline \multicolumn{15}{|l|}{ Gender } \\
\hline Male & 76 & 354 & 339 & 364 & 372 & 366 & 351 & 386 & 382 & 357 & 387 & 340 & 405.4 & $<0.0001$ \\
\hline Female & 108 & 538 & 516 & 526 & 536 & 491 & 480 & 506 & 499 & 470 & 480 & 468 & 343.4 & $<0.0001$ \\
\hline \multicolumn{15}{|l|}{ Race } \\
\hline White & 113 & 516 & 491 & 513 & 541 & 508 & 458 & 550 & 580 & 561 & 585 & 492 & 418.2 & $<0.0001$ \\
\hline Black & 15 & 81 & 76 & 83 & 66 & 78 & 83 & 93 & 82 & 107 & 117 & 80 & 662.6 & $<0.0001$ \\
\hline Hispanic & 10 & 52 & 53 & 39 & 50 & 57 & 50 & 48 & 61 & 51 & 57 & 48 & 497.6 & $<0.0001$ \\
\hline Others & 3 & 18 & 22 & 23 & 22 & 25 & 22 & 31 & 34 & 28 & 29 & 23 & 823.1 & $<0.0001$ \\
\hline \multicolumn{15}{|l|}{ Region } \\
\hline Northeast & 42 & 204 & 186 & 181 & 204 & 185 & 170 & 183 & 175 & 179 & 182 & 172 & 339.1 & $<0.0001$ \\
\hline Midwest & 45 & 195 & 221 & 235 & 217 & 209 & 229 & 222 & 228 & 207 & 228 & 203 & 404.1 & $<0.0001$ \\
\hline South & 72 & 349 & 313 & 338 & 339 & 325 & 304 & 347 & 351 & 327 & 327 & 308 & 355.4 & $<0.0001$ \\
\hline West & 26 & 144 & 135 & 135 & 150 & 139 & 128 & 141 & 129 & 114 & 130 & 125 & 393.3 & $<0.0001$ \\
\hline \multicolumn{15}{|l|}{ Location } \\
\hline Rural & 25 & 113 & 136 & 101 & 108 & 85 & 94 & 99 & 108 & 95 & 82 & 95 & 227.9 & $<0.0001$ \\
\hline Urban nonteaching & 91 & 438 & 406 & 454 & 505 & 427 & 396 & 433 & 409 & 393 & 389 & 395 & 326.2 & $<0.0001$ \\
\hline Urban teaching & 68 & 340 & 312 & 334 & 296 & 344 & 337 & 360 & 353 & 332 & 387 & 315 & 465.5 & $<0.0001$ \\
\hline \multicolumn{15}{|l|}{ Median Household Income } \\
\hline Quartile 1 & 12 & 44 & 218 & 221 & 219 & 210 & 222 & 235 & 228 & 224 & 240 & 188 & 1938.5 & $<0.0001$ \\
\hline Quartile 2 & 44 & 174 & 229 & 240 & 230 & 211 & 220 & 245 & 237 & 214 & 212 & 205 & 382.6 & $<0.0001$ \\
\hline Quartile 3 & 49 & 224 & 212 & 209 & 239 & 217 & 197 & 210 & 212 & 203 & 224 & 200 & 359.0 & $<0.0001$ \\
\hline Quartile 4 & 78 & 430 & 180 & 204 & 205 & 204 & 178 & 191 & 189 & 174 & 179 & 201 & 128.9 & $<0.0001$ \\
\hline \multicolumn{15}{|l|}{ Payment } \\
\hline Medicare & 153 & 743 & 714 & 726 & 753 & 705 & 682 & 716 & 699 & 667 & 710 & 661 & 363.5 & $<0.0001$ \\
\hline Medicaid & 5 & 27 & 27 & 26 & 29 & 31 & 24 & 28 & 35 & 36 & 32 & 27 & 491.7 & $<0.0001$ \\
\hline Private insurance & 22 & 106 & 97 & 114 & 105 & 97 & 107 & 126 & 122 & 103 & 100 & 100 & 350.2 & $<0.0001$ \\
\hline Others (includes self-pay) & 4 & 16 & 16 & 24 & 21 & 22 & 18 & 23 & 25 & 21 & 22 & 19 & 457.1 & $<0.0001$ \\
\hline \multicolumn{15}{|l|}{ Bed size } \\
\hline Small & 20 & 94 & 86 & 83 & 95 & 107 & 87 & 100 & 100 & 83 & 99 & 87 & 401.3 & $<0.0001$ \\
\hline Medium & 49 & 226 & 219 & 234 & 222 & 223 & 232 & 212 & 199 & 200 & 213 & 203 & 337.6 & $<0.0001$ \\
\hline Large & 116 & 572 & 549 & 572 & 591 & 527 & 509 & 580 & 571 & 538 & 546 & 516 & 369.3 & $<0.0001$ \\
\hline
\end{tabular}

\section{AHRQ Co-morbidities}

As per our analysis, the most common diagnoses associated with ADOI hospitalizations were hypertension (53.5\%), renal failure (33.1\%), and diabetes mellitus (28\%). Trends of several other comorbidities associated with ADOI also increased significantly, as depicted in Table 1.

\section{All-cause Inpatient Mortality}

Overall in-hospital all-cause mortality associated with these hospitalizations was $2 \%$. Unsurprisingly, the overall mortality rate for patients hospitalized with ADOI with hemorrhage was higher than those without hemorrhage (2.2\% vs 1.6\%) (Figure 3). However, the mortality rate for ADOI with hemorrhage decreased from $2.3 \%$ in 2001 to $1.9 \%$ in 2011 $(19.2 \%$ decrease; $p<0.0001)$ whereas it increased from $1.5 \%$ to $2.3 \%$ for ADOI without hemorrhage during the study period (53\% increase; $p<0.0001$ ) (Table 3). The mortality rate was highest in $\geq 80$-year age group at $2.5 \%$. The mortality rate was higher in males (2.1\%) than females (1.9\%). Also, mortality rate was highest in West $(2.4 \%)$ and hospitalizations paid by Medicaid (2.2\%). There was no significant difference in mortality rate as far as bed size was concerned (Table 3). 


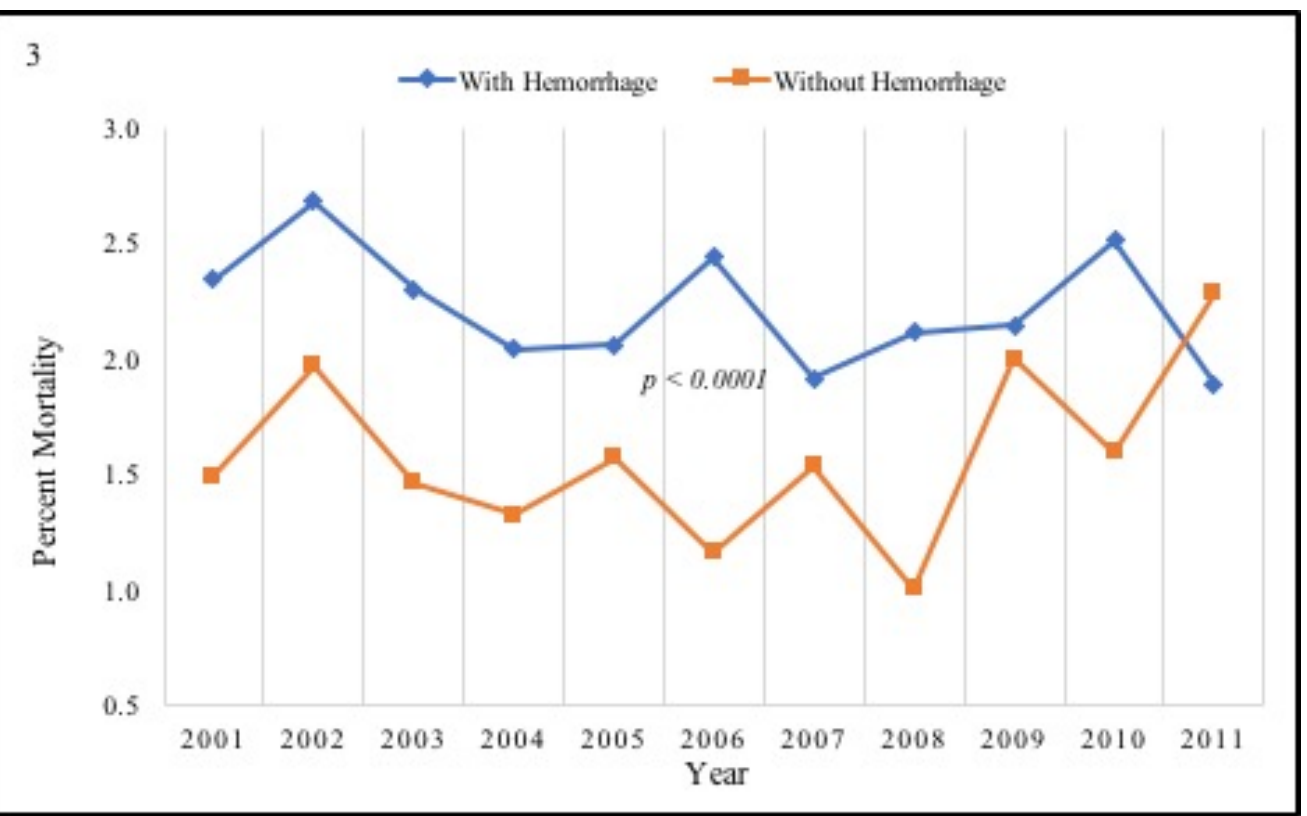

Figure 3: All-cause inpatient mortality rate in ADOI hospitalizations with and without hemorrhage

Table 3: In-hospital mortality (\%) for angiodysplasia hospitalizations

\begin{tabular}{|c|c|c|c|c|c|c|c|c|c|c|c|c|c|c|}
\hline Year & 2001 & 2002 & 2003 & 2004 & 2005 & 2006 & 2007 & 2008 & 2009 & 2010 & 2011 & Average & $\begin{array}{l}\text { Percent } \\
\text { change }\end{array}$ & $P$ value \\
\hline Overall & 2.1 & 2.5 & 2.0 & 1.8 & 1.9 & 2.0 & 1.8 & 1.7 & 2.1 & 2.1 & 2.1 & 2.0 & -1.0 & 0.02 \\
\hline \multicolumn{15}{|l|}{ Hemorrhage } \\
\hline Yes & 2.3 & 2.7 & 2.3 & 2.0 & 2.1 & 2.4 & 1.9 & 2.1 & 2.1 & 2.5 & 1.9 & 2.2 & -19.2 & $<0.0001$ \\
\hline No & 1.5 & 2.0 & 1.5 & 1.3 & 1.6 & 1.2 & 1.5 & 1.0 & 2.0 & 1.6 & 2.3 & 1.6 & 53.0 & $<0.0001$ \\
\hline \multicolumn{15}{|l|}{ Age in years } \\
\hline $18-34$ & 0.0 & 0.0 & 0.0 & 2.1 & 0.0 & 0.0 & 0.0 & 2.5 & 4.5 & 0.0 & 1.7 & 1.0 & - & 0.81 \\
\hline $35-49$ & 0.6 & 2.2 & 2.2 & 0.0 & 1.9 & 1.0 & 0.0 & 1.3 & 0.4 & 2.2 & 1.7 & 1.2 & 196.5 & $<0.0001$ \\
\hline 50-64 & 1.7 & 1.7 & 2.3 & 1.3 & 1.3 & 1.1 & 1.1 & 1.5 & 2.0 & 1.7 & 0.9 & 1.5 & -43.6 & $<0.0001$ \\
\hline $65-79$ & 2.0 & 1.8 & 1.7 & 1.4 & 1.5 & 1.7 & 1.6 & 1.5 & 1.9 & 2.2 & 2.0 & 1.7 & 2.1 & $<0.0001$ \\
\hline$>=80$ & 2.4 & 3.3 & 2.4 & 2.5 & 2.6 & 2.7 & 2.3 & 2.0 & 2.4 & 2.3 & 2.6 & 2.5 & 5.8 & $<0.0001$ \\
\hline \multicolumn{15}{|l|}{ Gender } \\
\hline Male & 2.2 & 2.9 & 2.0 & 2.1 & 2.4 & 1.9 & 1.8 & 2.2 & 2.0 & 2.3 & 1.9 & 2.1 & -13.9 & $<0.0001$ \\
\hline Female & 2.0 & 2.2 & 2.1 & 1.6 & 1.6 & 2.1 & 1.8 & 1.4 & 2.1 & 2.1 & 2.2 & 1.9 & 10.5 & $<0.0001$ \\
\hline \multicolumn{15}{|l|}{ Race } \\
\hline White & 2.3 & 2.4 & 2.0 & 1.8 & 2.2 & 2.0 & 1.7 & 1.7 & 2.1 & 2.0 & 2.2 & 2.0 & -3.5 & 0.48 \\
\hline Black & 2.7 & 2.8 & 2.7 & 1.8 & 1.4 & 1.9 & 2.0 & 1.4 & 2.1 & 2.5 & 1.3 & 2.0 & -51.3 & 0.26 \\
\hline Hispanic & 2.2 & 3.2 & 2.5 & 1.9 & 0.6 & 1.8 & 1.9 & 1.4 & 2.0 & 1.7 & 2.0 & 1.9 & -7.9 & 0.05 \\
\hline Others & 1.5 & 4.2 & 2.1 & 2.9 & 3.9 & 1.3 & 3.0 & 1.0 & 2.6 & 4.2 & 1.4 & 2.5 & -6.1 & 0.0002 \\
\hline \multicolumn{15}{|l|}{ Region } \\
\hline Northeast & 2.1 & 3.0 & 2.2 & 1.8 & 2.3 & 1.9 & 1.8 & 2.0 & 2.2 & 1.8 & 2.1 & 2.1 & 1.0 & $<0.0001$ \\
\hline Midwest & 1.9 & 2.0 & 1.7 & 1.6 & 2.3 & 2.0 & 1.6 & 1.7 & 2.0 & 2.3 & 1.9 & 1.9 & -0.5 & 0.008 \\
\hline South & 1.8 & 2.1 & 1.8 & 1.7 & 1.5 & 2.1 & 1.8 & 1.7 & 1.8 & 1.9 & 2.0 & 1.8 & 11.0 & $<0.0001$ \\
\hline West & 3.0 & 3.0 & 2.9 & 2.4 & 1.9 & 1.9 & 2.1 & 1.4 & 2.8 & 3.0 & 2.3 & 2.4 & -22.5 & $<0.0001$ \\
\hline \multicolumn{15}{|l|}{ Location } \\
\hline Rural & 2.6 & 2.5 & 1.7 & 2.1 & 1.9 & 1.7 & 1.9 & 1.3 & 1.8 & 1.7 & 2.5 & 2.0 & -3.5 & 0.32 \\
\hline Urban nonteaching & 1.9 & 2.2 & 2.3 & 1.6 & 1.9 & 2.1 & 1.9 & 1.9 & 2.1 & 1.9 & 2.3 & 2.0 & 19.2 & 0.33 \\
\hline Urban teaching & 2.1 & 2.8 & 1.9 & 2.0 & 1.9 & 1.9 & 1.7 & 1.6 & 2.3 & 2.5 & 1.7 & 2.0 & -17.1 & 0.25 \\
\hline \multicolumn{15}{|l|}{ Median Household Income } \\
\hline Quartile 1 & 2.4 & 2.1 & 1.8 & 1.6 & 2.0 & 1.9 & 2.2 & 1.5 & 1.6 & 2.5 & 2.3 & 2.0 & -3.8 & 0.05 \\
\hline Quartile 2 & 2.5 & 2.2 & 2.4 & 1.8 & 1.7 & 1.9 & 1.5 & 1.9 & 2.2 & 1.9 & 2.0 & 2.0 & -19.8 & 0.41 \\
\hline Quartile 3 & 2.0 & 2.8 & 1.9 & 1.8 & 2.5 & 2.5 & 1.8 & 1.5 & 1.6 & 2.3 & 2.0 & 2.1 & 0.5 & 0.05 \\
\hline Quartile 4 & 1.8 & 2.4 & 2.0 & 2.0 & 1.6 & 1.7 & 1.6 & 2.0 & 2.9 & 1.9 & 1.8 & 2.0 & 1.1 & 0.48 \\
\hline \multicolumn{15}{|l|}{ Payment } \\
\hline Medicare & 2.2 & 2.5 & 2.0 & 2.0 & 2.0 & 2.1 & 1.9 & 1.8 & 2.2 & 2.2 & 2.3 & 2.1 & 7.4 & $<0.0001$ \\
\hline Medicaid & 2.8 & 3.6 & 4.0 & 2.7 & 1.6 & 3.0 & 1.8 & 1.2 & 1.6 & 1.1 & 1.0 & 2.2 & -65.0 & 0.14 \\
\hline Private insurance & 1.5 & 2.0 & 1.9 & 0.7 & 1.6 & 1.2 & 1.2 & 1.5 & 1.6 & 2.0 & 0.8 & 1.5 & -44.8 & $<0.0001$ \\
\hline Others (includes self-pay) & 1.2 & 1.9 & 3.4 & 1.0 & 1.5 & 1.4 & 2.4 & 1.0 & 3.0 & 2.6 & 0.9 & 1.9 & -24.4 & 0.07 \\
\hline \multicolumn{15}{|l|}{ Bed size } \\
\hline Small & 1.2 & 3.8 & 1.4 & 2.3 & 2.6 & 1.8 & 1.5 & 1.7 & 2.0 & 2.1 & 2.0 & 2.0 & 65.6 & 0.29 \\
\hline Medium & 2.4 & 2.7 & 2.2 & 1.8 & 1.7 & 1.8 & 2.0 & 1.7 & 1.6 & 2.2 & 1.9 & 2.0 & -20.3 & 0.45 \\
\hline$\underline{\text { Large }}$ & 2.1 & 2.1 & 2.1 & 1.8 & 1.9 & 2.1 & 1.7 & 1.7 & 2.3 & 2.1 & 2.1 & 2.0 & 1.4 & 0.50 \\
\hline
\end{tabular}


Table 4: Cost (in U.S. dollars) of angiodysplasia hospitalizations

\begin{tabular}{|c|c|c|c|c|c|c|c|c|c|c|c|c|c|c|}
\hline Year & 2001 & 2002 & 2003 & 2004 & 2005 & 2006 & 2007 & 2008 & 2009 & 2010 & 2011 & Average & $\begin{array}{l}\text { Percent } \\
\text { Change }\end{array}$ & $P$ value \\
\hline Number of obs. (n) & 39,244 & 38,297 & 37,122 & 39,048 & 40,336 & 38,518 & 37,749 & 41,068 & 41,029 & 38,827 & 41,214 & 39,314 & 5.0 & $<0.0001$ \\
\hline Average cost (\$) & 10,701 & 11,530 & 11,491 & 11,422 & 12,064 & 11,715 & 11,607 & 11,945 & 12,323 & 13,337 & 12,478 & 11,874 & 16.6 & \\
\hline $\begin{array}{l}\text { Total cost per year (in } \\
\text { millions) }\end{array}$ & 420 & 442 & 427 & 446 & 487 & 451 & 438 & 491 & 507 & 518 & 514 & 467 & 22.5 & \\
\hline \multicolumn{15}{|l|}{ Age in years } \\
\hline $18-34$ & 165 & 180 & 221 & 295 & 216 & 191 & 200 & 217 & 195 & 295 & 273 & 223 & 65.3 & $<0.0001$ \\
\hline $35-49$ & 977 & 883 & 869 & 1,044 & 1,068 & 980 & 918 & 1,078 & 1,224 & 1,086 & 820 & 995 & -16.0 & 0.127 \\
\hline $50-64$ & 4,016 & 4,174 & 4,251 & 4,539 & 5,035 & 5,150 & 4,426 & 5,467 & 5,726 & 5,310 & 6,288 & 4,944 & 56.6 & $<0.0001$ \\
\hline $65-79$ & 17,791 & 17,222 & 17,044 & 17,262 & 17,349 & 16,558 & 16,626 & 17,147 & 17,426 & 16,735 & 17,120 & 17,116 & -3.8 & $<0.0001$ \\
\hline$>=80$ & 16,280 & 15,786 & 14,713 & 15,861 & 16,590 & 15,583 & 15,547 & 17,101 & 16,432 & 15,370 & 16,694 & 15,996 & 2.5 & 0.0008 \\
\hline \multicolumn{15}{|l|}{ Gender } \\
\hline Male & 16,238 & 15,194 & 14,732 & 15,961 & 16,536 & 16,454 & 15,959 & 17,775 & 17,785 & 16,756 & 18,372 & 16,524 & 13.1 & $<0.0001$ \\
\hline Female & 23,007 & 23,103 & 22,390 & 23,087 & 23,794 & 22,065 & 21,790 & 23,288 & 23,240 & 22,072 & 22,837 & 22,788 & -0.7 & $<0.0001$ \\
\hline \multicolumn{15}{|l|}{ Race } \\
\hline White & 23,970 & 22,169 & 21,317 & 22,520 & 24,036 & 22,805 & 20,818 & 25,280 & 26,966 & 26,324 & 27,810 & 24,001 & 16.0 & $<0.0001$ \\
\hline Black & 3,261 & 3,484 & 3,301 & 3,640 & 2,910 & 3,495 & 3,773 & 4,271 & 3,823 & 5,017 & 5,568 & 3,867 & 70.7 & $<0.0001$ \\
\hline Hispanic & 2,033 & 2,224 & 2,295 & 1,701 & 2,225 & 2,578 & 2,269 & 2,229 & 2,833 & 2,374 & 2,720 & 2,316 & 33.8 & 0.4758 \\
\hline Others & 657 & 768 & 961 & 1,000 & 987 & 1,127 & 1,020 & 1,416 & 1,591 & 1,307 & 1,358 & 1,108 & 106.7 & $<0.0001$ \\
\hline \multicolumn{15}{|l|}{ Region } \\
\hline Northeast & 8,819 & 8,745 & 8,086 & 7,964 & 9,038 & 8,296 & 7,729 & 8,425 & 8,126 & 8,397 & 8,670 & 8,390 & -1.7 & $<0.0001$ \\
\hline Midwest & 9,604 & 8,360 & 9,574 & 10,317 & 9,624 & 9,370 & 10,385 & 10,191 & 10,589 & 9,705 & 10,840 & 9,869 & 12.9 & $<0.0001$ \\
\hline South & 15,246 & 14,996 & 13,585 & 14,857 & 15,032 & 14,588 & 13,804 & 15,965 & 16,314 & 15,362 & 15,545 & 15,027 & 2.0 & $<0.0001$ \\
\hline West & 5,576 & 6,196 & 5,876 & 5,910 & 6,642 & 6,265 & 5,831 & 6,488 & 6,001 & 5,363 & 6,158 & 6,028 & 10.5 & $<0.0001$ \\
\hline \multicolumn{15}{|l|}{ Location } \\
\hline Rural & 5,339 & 4,867 & 5,900 & 4,453 & 4,805 & 3,800 & 4,293 & 4,551 & 5,026 & 4,472 & 3,919 & 4,675 & -26.6 & $<0.0001$ \\
\hline Urban nonteaching & 19,374 & 18,816 & 17,638 & 19,950 & 22,405 & 19,192 & 18,017 & 19,929 & 19,025 & 18,437 & 18,487 & 19,206 & -4.6 & $<0.0001$ \\
\hline Urban teaching & 14,532 & 14,614 & 13,537 & 14,645 & 13,126 & 15,474 & 15,319 & 16,573 & 16,417 & 15,592 & 18,398 & 5,293 & 26.6 & .0064 \\
\hline $\begin{array}{l}\text { Median Household } \\
\text { Income }\end{array}$ & & & & & & & & & & & & & & \\
\hline Quartile 1 & 2,496 & 1,889 & 9,442 & 9,697 & 9,708 & 9,440 & 10,086 & 10,805 & 10,621 & 10,527 & 11,393 & 8,737 & 356.4 & $<0.0001$ \\
\hline Quartile 2 & 9,313 & 7,464 & 9,954 & 10,525 & 10,199 & 9,477 & 9,981 & 11,277 & 11,044 & 10,016 & 10,061 & 9,938 & 8.0 & $<0.0001$ \\
\hline Quartile 3 & 10,368 & 9,622 & 9,203 & 9,172 & 10,593 & 9,758 & 8,966 & 9,672 & 9,886 & 9,517 & 10,656 & 9,765 & 2.8 & $<0.0001$ \\
\hline Quartile 4 & 16,607 & 18,482 & 7,819 & 8,974 & 9,112 & 9,144 & 8,109 & 8,767 & 8,789 & 8,156 & 8,510 & 10,225 & -48.8 & $<0.0001$ \\
\hline \multicolumn{15}{|l|}{ Payment } \\
\hline Medicare & 32,507 & 31,899 & 30,980 & 31,854 & 33,430 & 31,675 & 30,977 & 32,915 & 32,515 & 31,269 & 33,737 & 32,160 & 3.8 & $<0.0001$ \\
\hline Medicaid & 1,143 & 1,151 & 1,192 & 1,158 & 1,303 & 1,389 & 1,082 & 1,295 & 1,624 & 1,707 & 1,514 & 1,323 & 32.5 & $<0.0001$ \\
\hline Private insurance & 4,719 & 4,546 & 4,220 & 4,996 & 4,673 & 4,367 & 4,873 & 5,811 & 5,664 & 4,830 & 4,756 & 4,860 & 0.8 & $<0.0001$ \\
\hline $\begin{array}{l}\text { Others (includes self- } \\
\text { pay) }\end{array}$ & 850 & 691 & 674 & 6 & 929 & 999 & 798 & 1,037 & 1,147 & 968 & 1,061 & 926 & 24.7 & 001 \\
\hline \multicolumn{15}{|l|}{ Bed size } \\
\hline Small & 4,198 & 4,026 & 3,728 & 3,635 & 4,196 & 4,808 & 3,975 & 4,598 & 4,641 & 3,874 & 4,712 & 4,217 & 12.2 & $<0.0001$ \\
\hline Medium & 10,341 & 9,710 & 9,502 & 10,288 & 9,878 & 9,998 & 10,522 & 9,765 & 9,236 & 9,389 & 10,132 & 9,887 & -2.0 & $<0.0001$ \\
\hline Large & 24,705 & 24,561 & 23,846 & 25,125 & 26,262 & 23,661 & 23,133 & 26,690 & 26,590 & 25,238 & 25,960 & 25,070 & 5.1 & $<0.0001$ \\
\hline
\end{tabular}

\section{Length of Stay and Cost of Care}

Median length of stay (LOS) was 4 days (interquartile range 2 to 7 days). The mean cost of care increased from $\$ 10,701$ per hospitalization in 2001 to $\$ 12,478$ in 2011 (16.6\% increase; $p<0.0001$ ) (Figure 4). The total cost of all such hospitalizations increased from $\$ 419$ million in 2001 to $\$ 514$ million in 2011 (Table 4). 


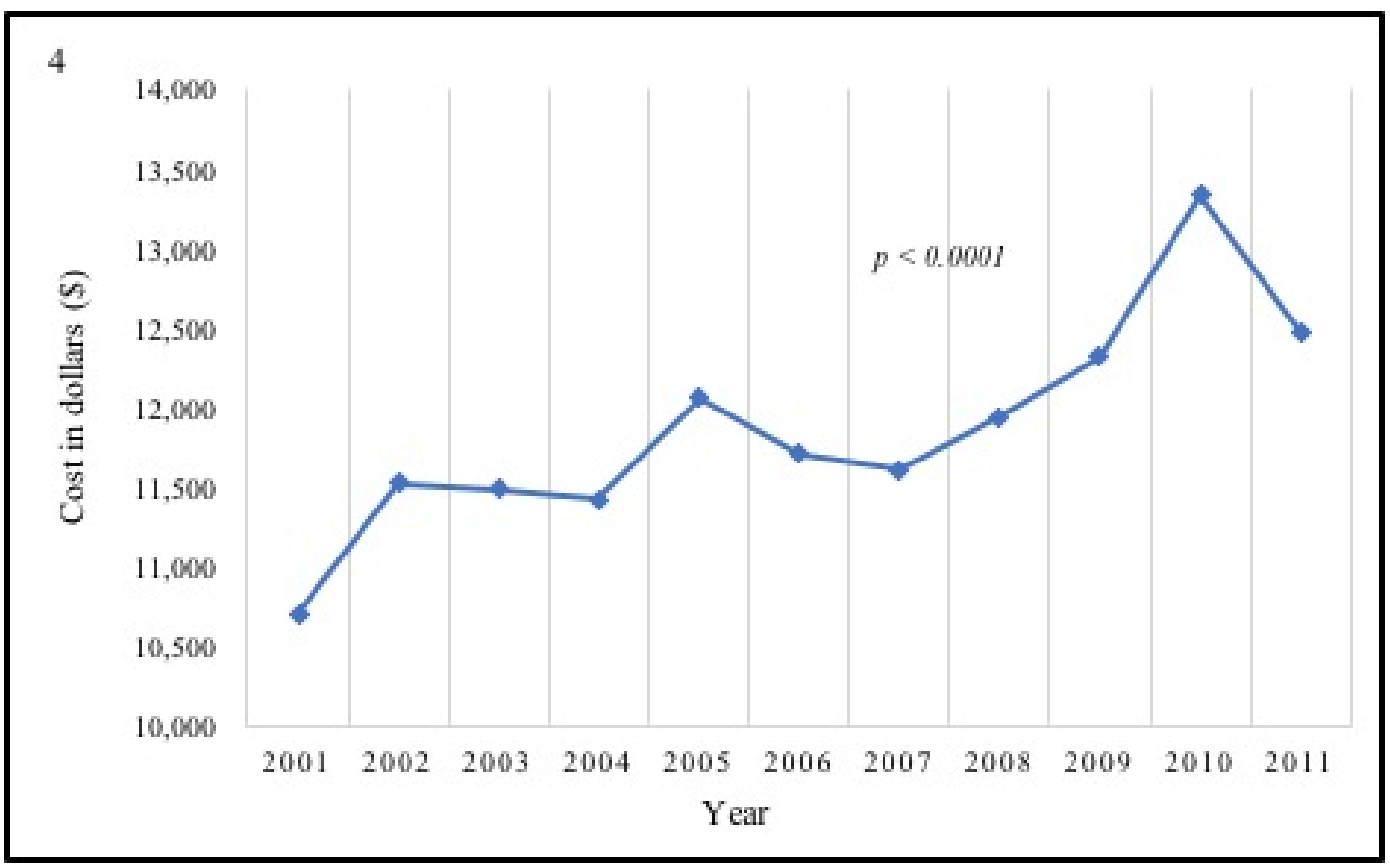

Figure 4: Average cost of care for ADOI related hospitalizations

\section{DISCUSSION}

Our study reports important findings associated with ADOI over a 10-year period in the U.S. Angiodysplasia accounts for a large percentage of GI bleeding, however, as they are commonly asymptomatic and found during investigation of an unrelated issue, limited epidemiologic information is available on the subject (2). We found that the number of hospitalizations related to angiodysplasia, both with and without hemorrhage, significantly increased during the study period. Cost of care per hospitalization related to this diagnosis increased by a staggering $\$ 95$ million dollars over the course of the study period.

The etiology of angiodysplasias is not fully understood. Recent theory suggests that the lesions are the result of dilated and tortuous veins in the submucosa due to chronic low-grade intermittent obstruction of submucosal veins (13).

This idea is supported by the finding that angiodysplasias are most commonly located in areas of the GI tract with of high wall tension including the cecum and gastric antrum. It is not surprising then that the majority of angiodysplasia are acquired with age (3). Consistent with previously published studies, most of the hospitalizations in our study were identified in the 65 to 74 year age group $(14,15)$. Survival bias and other factors such as multiple comorbidities, must also be considered as possible explanations of this trend.

From our analysis of the comorbidity measures, we found that the most frequent co-existing conditions were hypertension, diabetes, and renal failure. Von Willebrand disease and aortic stenosis have also been linked to higher rates of angiodysplasia. However, it remains unclear if it is the incidence or just detection rates in these populations that are higher (16). Patients who have bled from gastrointestinal angiodysplasia and aortic stenosis should be referred for an evaluation for aortic valve replacement (17).

Since the clinical presentation of angiodysplasia displays considerable variation, ranging from asymptomatic occult blood loss to life threatening Gl hemorrhage, it is often difficult to diagnose (2). More recently, expanded use of capsule endoscopy, balloon-assisted enteroscopy and computed tomographic angiography have enabled care providers to improve their diagnostic capabilities $(17,20)$. Several treatment options including endoscopic interventions, endovascular embolization and surgery including minimally invasive procedures exist (21). Interestingly, biomarkers such as Ang-2 and TIE-2 may now be able to predict the presence of gastrointestinal angiodysplasia and predict targets for future therapies (17). Hence, facilities with highly trained specialists utilizing more sophisticated equipment are often required for diagnosis and management. Therefore, it is not surprising that hospitalizations with ADOI were found to be greater in larger more urban centers.

Throughout the study period, more women than men were hospitalized with a diagnosis of ADOI. However, our analysis revealed a change in trends; the percent increase in hospitalizations during the study period was higher in men than women. Hospitalizations were also higher in the white population, with the highest percent increase in 
hospitalizations per million in the Midwest region. Hernandez et al recently published a study in which they found a large geographic variation in oral anticoagulation use for atrial fibrillation, with the highest rates in the Midwest region of the U.S (22). Increased bleeding risk in this population might explain a portion of the percent increased rate of hospitalizations for ADOI in this region.

Overall, in-hospital all-cause mortality associated with these hospitalizations was $2 \%$. Not surprisingly, mortality rates were highest in the $>80$-year age group. Although mortality rates were higher in those with ADOI with hemorrhage, the mortality rate for ADOI with hemorrhage decreased during the study period. We postulate this is the result of advancements in imaging and endoscopic techniques enhancing our ability to better detect and manage occult gastrointestinal bleeding through the Gl tract. Additionally, studies have shown newer techniques such as capsule endoscopy and double-balloon enteroscopy have a high diagnostic yield and positive impact on management in patients aged 65-70 years or more $(23,25)$. Endotherapy with argon plasma coagulation resolves recurrent bleeding episodes in $83 \%$ of patients with $\mathrm{Gl}$ angiodysplasias (26). In patients who fail (or are not suitable for) other interventions, treatment with thalidomide or octreotide can lead to a clinically meaningful response in $71.4 \%$ and $77 \%$ of patients respectively (2).

This study found a significant increase in the cost of care for ADOI related hospitalizations from 2001 to 2011. Greater use of advanced diagnostic and therapeutic techniques may be a major factor driving up costs. Alternatively, improvements in coding accuracy may explain this rising trend.

Analysis of the NIS database permitted us to study a large population. Such analysis should decrease the inherent bias seen in studies that are confined to a single region or hospital (4). However; there are limitations when it comes to using this type of healthcare utilization database. Multiple factors such as coding inaccuracies, missing clinical information, and the lack of outpatient characteristics, increase the potential for both overestimating and underestimating a particular diagnosis.

For example, our analysis of the NIS database using the ICD-9 codes did not permit us to differentiate between new onset versus prior history of hospitalization for ADOI with bleeding. In addition, the NIS considers each hospitalization as a separate entry; therefore, it is not possible to separate a new case of ADOI from a readmission (7). These factors could result in an overestimation in the number of cases. Conversely, as angiodysplasias are often the culprit behind occult bleeding, particularly in the non-hospitalized population, there is a risk that our analysis could underestimate the number of bleeds related to angiodysplasia each year. Also important to consider is the fact that the lack of detailed clinical information may result in misclassification of outcomes related to a diagnosis (27).

Using the NIS database, we were only able to examine in-hospital characteristics; as such, the study did not permit analysis of long-term follow up of patient outcomes. In addition, it did not permit us to study health care utilization in out-patient settings or emergency departments.

In conclusion, our analysis of hospitalization trends from 2001-2011, found a consistent increase in the number of hospitalizations associated with angiodysplasia of the intestine as well as the cost of associated care. Angiodysplasia is now recognized as a major cause of both occult and overt Gl bleeding (28). We believe that increases in awareness of the condition, along with improved diagnostic and therapeutic techniques, may result in further improvements in mortality rates in the future. Whether this will reduce the economic burden will require further study, but could be favorably influenced by efforts to ensure early diagnosis and to make treatment available at more health care centers.

\section{REFERENCES}

1. Regula J, Wronska E, Pachlewski J. Vascular lesions of the gastrointestinal tract. Best Pract Res Clin Gastroenterol 2008;22(2):313-28. https://doi.org/10.1016/j.bpg.2007.10.026. PMid:18346686

2. Sami SS, Al-Araji SA, Ragunath K. Review article: gastrointestinal angiodysplasia - pathogenesis, diagnosis and management. Aliment Pharmacol Ther. 2014;39(1):15-34. https://doi.org/10.1111/apt.12527. PMid:24138285

3. Foutch PG. Angiodysplasia of the gastrointestinal tract. Am J Gastroenterol 1993, 88(6):807-18. PMid:8389094

4. Overview of the National (Nationwide) Inpatient Sample (NIS). Retrieved on 17 January 2018 from https://www.hcup-us.ahrq.gov/nisoverview.jsp

5. NIS Related Reports. Retrieved on 17 January 2018 from https://www.hcup-us.ahrq.gov/db/nation/nis/ nisrelatedreports.jsp

6. Badheka AO, Chothani A, Mehta K, et al. Utilization and adverse outcomes of percutaneous left atrial appendage closure for stroke prevention in atrial fibrillation in the United States: influence of hospital volume. Circ Arrhythm Electrophysiol. 2015;8(1):42-8. https://doi.org/10.1161/CIRCEP.114.001413 PMid:25480543 
7. Patel NJ, Deshmukh A, Pant $\mathrm{S}$, et al. Contemporary trends of hospitalization for atrial fibrillation in the United States, 2000 through 2010: implications for healthcare planning. Circulation 2014;129(23):2371-9. https://doi.org/10.1161/CIRCULATIONAHA.114.008201 PMid:24842943

8. Singh V, Rodriguez AP, Thakkar B, et al. Hospital Admissions for Chest Pain Associated with Cocaine Use in the United States. Am J Med. 2017;130(6):688-98. https://doi.org/10.1016/j.amjmed.2016.12.003 PMid:28063854

9. Population and Housing Unit Estimates. Retrieved on 18 January 2018 from https://www.census.gov/programssurveys/popest.html

10. HCUP Methods Series. Retrieved on 17 January 2018 from https://www.hcup-us.ahrq.gov/reports/methods/ 1998ChangesintheNISRedesignFinal.pdf

11. Armitage P: Tests for Linear Trends in Proportions and Frequencies. Biometrics 1955;11(3):375-386. https://doi.org/10.2307/3001775

12. Nahm FS: Nonparametric statistical tests for the continuous data: the basic concept and the practical use. Korean J Anesthesiol 2016;69(1):8-14. https://doi.org/10.4097/kjae.2016.69.1.8 PMid:26885295 PMCid:PMC4754273

13. Boley SJ, Sammartano R, Adams $A$, et al. On the nature and etiology of vascular ectasias of the colon. Degenerative lesions of aging. Gastroenterology 1977;72(4 Pt 1):650-60. PMid:300063

14. Clouse RE, Costigan DJ, Mills BA, et al. Angiodysplasia as a cause of upper gastrointestinal bleeding. Arch Intern Med. 1985;145(3):458-61. https://doi.org/10.1001/archinte.145.3.458 PMid:3872107

15. Richter JM, Hedberg SE, Athanasoulis CA, et al. Angiodysplasia. Clinical presentation and colonoscopic diagnosis. Dig Dis Sci. 1984;29(6):481-5. https://doi.org/10.1007/BF01296266 PMid:6609803

16. Cappell MS. Spatial clustering of simultaneous nonhereditary gastrointestinal angiodysplasia. Small but significant correlation between nonhereditary colonic and upper gastrointestinal angiodysplasia. Dig Dis Sci. 1992;37(7):1072-7. https://doi.org/10.1007/BF01300289 PMid:1618055

17. Jackson CS, Strong R. Gastrointestinal Angiodysplasia: Diagnosis and Management. Gastrointest Endosc Clin N Am. 2017;27(1):51-62. https://doi.org/10.1016/j.giec.2016.08.012 PMid:27908518

18. Godeschalk MF, Mensink PB, van Buuren HR, et al. Primary balloon-assisted enteroscopy in patients with obscure gastrointestinal bleeding: findings and outcome of therapy. J Clin Gastroenterol. 2010;44(9):e195-200. https://doi.org/10.1097/MCG.0b013e3181dd1110 PMid:20505527

19. Prachayakul V, Deesomsak M, Aswakul $P$, et al. The utility of single-balloon enteroscopy for the diagnosis and management of small bowel disorders according to their clinical manifestations: a retrospective review. BMC Gastroenterol. 2013;13:103. https://doi.org/10.1186/1471-230X-13-103 PMid:23800178 PMCid:PMC3716953

20. Pandey $V$, Ingle $M$, Pandav $N$, et al. The role of capsule endoscopy in etiological diagnosis and management of obscure gastrointestinal bleeding. Intest Res. 2016;14(1):69-74. https://doi.org/10.5217/ir.2016.14.1.69 PMid:26884737 PMCid:PMC4754525

21. Shah AR, Jala V, Arshad H, et al. Evaluation and management of lower gastrointestinal bleeding. Dis Mon 2018. https://doi.org/10.1016/j.disamonth.2018.02.002 PMid:29525374

22. Hernandez I, Saba S, Zhang Y. Geographic Variation in the Use of Oral Anticoagulation Therapy in Stroke Prevention in Atrial Fibrillation. Stroke 2017;48(8):2289-91. https://doi.org/10.1161/STROKEAHA.117.017683 PMid:28655815

23. Urgesi R, Cianci R, Pagliari $D$, et al. Is capsule endoscopy appropriate for elderly patients? The influence of ageing on findings and diagnostic yield: An Italian retrospective study. Dig Liver Dis. 2015;47(12):1086-8. https://doi.org/10.1016/j.dld.2015.09.004 PMid:26514739

24. Sidhu R, Sanders DS. Double-balloon enteroscopy in the elderly with obscure gastrointestinal bleeding: safety and feasibility. Eur J Gastroenterol Hepatol. 2013;25(10):1230-4. https://doi.org/10.1097/ MEG.0b013e3283630f1b

25. Li L, Chen C, Li Y, et al. The role of capsule endoscopy in the diagnosis and treatment of obscure gastrointestinal bleeding in older individuals. Eur J Gastroenterol Hepatol. 2016;28(12):1425-30. https://doi.org/10.1097/MEG.0000000000000737 PMid:27603298

26. Olmos JA, Marcolongo $M$, Pogorelsky $V$, et al. Argon plasma coagulation for prevention of recurrent bleeding from GI angiodysplasias. Gastroint. Endosc. 2004;60(6):881-6. https://doi.org/10.1016/S0016-5107(04)02221-7 
27. Schneeweiss S, Avorn J. A review of uses of health care utilization databases for epidemiologic research on therapeutics. J Clin Epidemiol. 2005;58(4):323-37. https://doi.org/10.1016/j.jclinepi.2004.10.012 PMid:15862718

28. Liao Z, Gao R, Xu C, et al. Indications and detection, completion, and retention rates of small-bowel capsule endoscopy: a systematic review. Gastrointest Endosc. 2010;71(2):280-6. https://doi.org/10.1016/ j.gie.2009.09.031 PMid:20152309

$\diamond \diamond \diamond \diamond \diamond \diamond \diamond$

http://www.ejgm.co.uk 